\title{
CHANNEL ASSIGNMENT IN AN IEEE 802.11 WLAN BASED ON SIGNAL-TO- INTERFERENCE RATIO
}

\author{
Mohamad Haidar $^{\# 1}$, Rabindra Ghimire ${ }^{\# 1}$, Hussain Al-Rizzo ${ }^{\# 1}$, Robert Akl ${ }^{\# 2}$, Yupo Chan ${ }^{\# 1}$ \\ ${ }^{\# 1}$ Department of Applied Science, University of Arkansas at Little Rock \\ 2801 S. University Ave., Little Rock, Arkansas, 72204, USA \\ \{mxhaidar, rxghimire, hmalrizzo, yxchan\} Qualr.edu

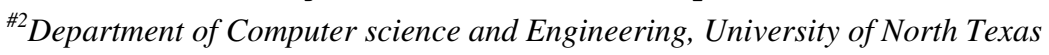 \\ 3940 N. Elm St., Denton, Texas, 76207, USA \\ rakl@cse.unt.edu
}

\begin{abstract}
In this paper, we propose a channel-assignment algorithm at the Access Points (APs) of a Wireless Local Area Network (WLAN) in order to maximize Signal-to-Interference Ratio (SIR) at the user level. We start with the channel assignment at the APs, which is based on minimizing the total interference between APs. Based on this initial assignment, we calculate the SIR for each user. The algorithm can be applied to any WLAN, irrespective of the user distribution and user load. Results show that the proposed algorithm is capable of significantly increasing the SIR over the WLAN, which in turn improves throughput.
\end{abstract}

Index Terms-Signal-to-Interference Ratio, WLAN, Channel Assignment, Access Points.

\section{INTRODUCTION}

Channel assignment in IEEE 802.11 WLAN has received significant attention in the past few years [1]-[5]. The increase in deployment of access points (APs) has led researchers to develop channel assignment algorithms in order to reduce cochannel and adjacent channel interferences from neighboring APs, which causes an overall throughput degradation of the network.

The authors in [1] proposed an approach for optimizing channel assignment of hot-spot service areas in a WLAN by formulating an Integer Linear Program (ILP). Their objective was to minimize the maximum channel utilization, thus equalizing the load distribution. This results in a higher throughput, through assigning non-overlapping channels among neighboring APs. In [2], the authors noted that previous AP placement and channel assignment were always designed sequentially. Therefore, they proposed an integrated model that addresses both at the same time. They showed that, by combining AP placement and channel assignment, the results were superior. A dynamic channel-assignment ILP that minimizes channel interference between neighboring APs at a reference AP is presented [3]. The channel assignment was done at the initial phase of planning, setting asideuser considerations. The authors in [4] applied the concept of channel assignment in the outdoor environment to the indoor environment. They installed three IEEE 802.11 compliant APs in an indoor environment and performed signal measurements to assign channels for the APs. An ILP then assigns channels to APs. Finally, the authors in [5] propose a weighted variant of the coloring graph algorithm to improve the usage of wireless spectrum in WLANs. The authors emphasized that a least congested channel assignment is not efficient with the continued growth of WLANs.

In this paper, we extend our work presented in [6] by proposing a mathematical model to assign channels to the APs based on maximizing the total SIR at the user level. It has been shown that SIR is directly proportional to the network throughput [7]. Therefore, improving the total SIR level over the network will lead to better data rate throughput over the network. Channel assignment is performed in two steps. After the network achieves a balanced state for balanced load distribution [6], we use the SIR of all users to reassign channels to the APs. The algorithm in [6] deals with distributing the load more efficiently among APs by reassigning users to different APs while decrementing the transmitted power of the Most Congested AP (MCAP). The current paper goes one step further to reassign channels based on SIR.

All related work that we are aware of to date has always considered minimizing the interference between neighboring APs. This is an efficient channel assignment if users are located exclusively in the overlap coverage region of the APs. In this case, minimizing interference between APs is the same as maximizing SIR for users. In reality, however, users distribute themselves at different locations in the study area. It should be emphasized that, to best of the authors' knowledge, the current paper is the first attempt to consider assigning channels to APs based on maximizing the SIR at the user level, which quantitatively leads to increase in network throughput as well as the channel reuse factor in some cases.

The remainder of this paper is organized as follows: The overlapping channel interference factor is reviewed in section II. In section III, we define our channel assignment model and algorithm. Numerical results are presented in section IV, and finally section $\mathrm{V}$ concludes the paper. 


\section{OVERLAPPING CHANNEL INTERFERENCE}

In IEEE $802.11 \mathrm{~b} / \mathrm{g}$ WLAN, there exist 14 channels. Channels 1, 6 and 11 are non-overlapping, as shown in Fig. 1. Of the 14 channels, only 11 are used in the US.

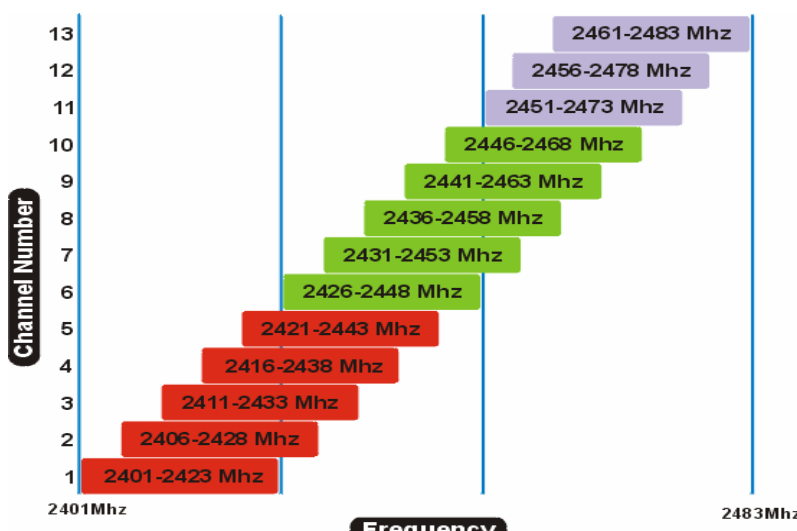

[8]

Fig. 1. The three non-overlapping channels (red, green and purple)

Each channel spreads over $22 \mathrm{MHz}$ due to the Direct Sequence Spread Spectrum (DSSS) technique employed by IEEE $802.11 \mathrm{~b} / \mathrm{g}$. For instance, channel 1 ranges from 2.401 $\mathrm{GHz}$ to $2.423 \mathrm{GHz}$ and its center frequency is $2.412 \mathrm{GHz}$. The center frequency of two adjacent channels is separated by 5 MHz. Therefore, there is channel bandwidth overlap. The interference-level factor $w_{j k}$ is defined as follows:

$$
w_{j k}=\max \left(0,1-\left|C h_{j}-C h_{k}\right| \times c\right)
$$

where $C h_{j}$ is the channel assigned to $A P_{j}, C h_{k}$ is the channel assigned to $A P_{k}$ and $c$ is the non-overlapping portion of two adjacent channels, expressed as a fraction of the frequency spectrum of a channel. For instance, channel 1 and channel 2 do not overlap from $2.401 \mathrm{GHz}$ to $2.406 \mathrm{GHz}$, as shown in Fig. 1. Normalizing the overlap of $5 \mathrm{MHz}$ over the spectrum of 23 $\mathrm{MHz}, c$ is equal to $1 / 5$ approximately. When the channels are far apart, as is the case with channels 1 and $6, w_{j k}=0$ (i.e., no interference). When the two channels are the same, $C h_{j}{ }^{\prime}-C h_{k}$ $=0$, Eqn 1 suggests that $w_{j k}=1$ (i.e., maximum interference). Therefore, channels should be assigned to APs such that overlapping channel interference is minimized. On the other hand, for channels 1 and $6,\left|C h_{j}{ }^{\prime}-C h_{k}\right|=5, w_{j k}=0$, suggesting no interference.

Mindful that we only have limited channel resources (11 channels in IEEE $802.11 \mathrm{~b} / \mathrm{g}$ ), some channels need to be "recycled." If the same channel is to be assigned to two or more APs which are located far enough from each others, the overlapping channel interference signal detected by each AP should be less than a given threshold.

\section{THE CHANNEL ASSIGNMENT MODEL}

Here, we will present a new channel-assignment model and algorithm for IEEE 802.11 WLAN systems. Channels should be assigned to each AP in such a way to maximize the SIR at the user level, rather than to minimize interference among APs. By maximizing the SIR of the whole user's network, the network channels' (resources) will be utilized more efficiently resulting in higher throughput.

We consider a WLAN consisting of $M$ APs situated in a single-floor service area. A set of randomly distributed $N$ users are to be served by these APs. Our algorithm is initiated by balancing the load based on power management algorithm [6]. Each user is assigned to one and only one AP at any time. This association assignment is assumed fixed.

The received power level for each user is evaluated using the No-Line-of-sight (NLOS) path-loss model in (2) [9].

$P L(d)=P L_{0}+29.4 \log _{10}(d)+6.1 x_{\alpha} \log _{10}(d)+24 y+1.3 x_{y} y$,

where $P L_{0}$ is the free space path loss, $d$ is the distance between user $i$ and $A P_{j}$, and $x_{a}, x_{s}$, and $y$ are mutually independent Gaussian random variables of zero mean and unit variance. These random variables model power loss due to factors other than distance.

We now formulate our channel-assignment problem as a Non-Linear Integer Program (NLIP) using the following variables defined below:

- $\quad \mathbf{A}_{\mathbf{j}}$ is the set of neighboring APs to $A P_{j}$.

- $K$ is the total number of available channels, 11 in IEEE $802.11 \mathrm{~b} / \mathrm{g}$.

- $\quad P_{i k}$ is the power received by user $i$ associated with $A P_{k}$.

- $\quad P_{i j}$ is the power received by user $i$ from the interfering $A P_{j}$.

- $\quad I_{i j}$ is the total interference experienced by user $i$ due to all APs $j$ (where $j \neq k$ ).

The channel assignment problem is modeled as (3):

$\max \sum_{i=1}^{N} \sum_{j=1}^{M} \operatorname{SIR}_{i j}(k), j \neq k$

subject to

$$
\begin{array}{r}
w_{j k}=\max \left(0,1-\left|C h_{j}-C h_{k}\right| \times c\right) \\
I_{i j}=\sum_{i=1}^{N} \sum_{j=1}^{M}\left(P_{i j} \bullet w_{j k}\right), \quad j \neq k \\
S I R_{i j}(k)=\frac{P_{i k}}{I_{i j}} \quad \forall i, j, j \neq k \\
j, k \in\{1, . ., M\} \\
i \in\{1, . . N\} \\
C h_{j}, C h_{k} \in\{1, . ., K\}
\end{array}
$$


Objective (3.1) maximizes the total SIR for all users $i$. Maximizing SIR is related to minimizing interference at the user level from neighboring APs which in turn, the interference at the user, is defined by the channel assignment. Constraint (3.2), a reproduction of (1) for convenience, defines the overlapping channel interference factor between $A P_{j}$ and $A P_{k}$, which have been assigned $C h_{j}$ and $C h_{k}$, respectively. Constraint (3.3) defines the interference experienced by user $i$ due to all APs except $A P_{k}$. Constraint (3.4) defines the signal-to-interference ratio for user $i$ due to interfering access points $j(j \neq k)$. The NLIP determines the best integer variables $C h_{j}$ and $C h_{k}$ or channel assignments that lead to the maximum SIR. This in turn results in the maximum throughput. It is observed that the non-linearity in the problem comes from the definition of the $w_{j k}$ variable, as shown in (3.2).

When executed in real time, we assume that each user $i$ updates his serving $\mathrm{AP}_{\mathrm{k}}$ with its associated $\operatorname{SIR}_{i}(k)=\sum_{j} \operatorname{SIR}_{i j}(k)$ upon registering with it. Then each AP, synchronized with the other APs, will periodically request SIR from its users. In case of a change in the current user distribution, resulting from users joining or leaving the network, the APs will transfer the $\operatorname{SIR}_{i j}(k)$ information to a central unit server that runs the channel-assignment model to reassign channels to the APs. All APs are assumed to be operated by the same internet service provider. However, the present scenarios in this paper do not involve user mobility. They are set up with a fixed number of APs, a fixed number of users, and a fixed data rate over the study period. The purpose of the displayed scenarios is to compare the effect of channel assignment at the initial design stage, in which the users have yet to enter the picture, and a later stage, when users are considered in the network.

One can think of our model as representing the scenario in a time slice, for a particular user distribution. It is important to note that user-to-user interference was assumed negligible due to its low transmitted power compared to the AP's transmitted power. The model can be executed for all time slices sequentially in which the $\operatorname{SIR}_{i j}(k)$ information is updated for each time slice.

Repeated execution of the NLIP model can be described by a number of computational steps. Such a channelassignment algorithm can be stated as follows:

1. Assign channels to the $M$ APs based on the NLIP model proposed in reference [3] which is based on minimizing the total interference between APs.

2. Input the positions of $N$ randomly distributed users.

3. Perform load balancing based on the power-management algorithm proposed in [6].

4. The model in [6] provides the received power by each user.

5. Compute interference caused by neighboring APs at each user.

6. Compute SIR for each user.

7. Run the NLIP model in equation (3).

8. Advance the time slice and go back to step 2 .
In theory, the above algorithm is executed ad infinitum. Let us assume continuity among time slides, in that states transition smoothly from one time slice to another. Also assume that the central unit server is fast enough to obtain current information, the algorithm can ensure efficient operation of a WLAN. To test this hypothesis, a simulation is run continuously until the balanced load state discussed in [6] is achieved among data based on existing user patterns. Because of the random distribution of the users, we ran more than 120 simulation replications for each scenario. It was judged that 120 replication cycles are sufficient to reach a steady state. During each replication cycle of the simulation, the association of user location $i$ to $\mathrm{AP}_{j}$ remain fixed-until a new association is obtained in step 3 we show the average results of each scenario below.

Instead of an optimization solver, the authors solved the problem in equation (3) by enumeration using Matlab software tool. The purpose of using an enumeration method is to gain some insight on the SIR value for each iteration. SIR values were examined closely until a maximum was obtained. The exercise will pave the way for a more formal optimization routine.

Note that the simulation time did not exceed 20 minutes each for all the scenarios presented. . However, the simulation time increases rapidly as the number of variables in (3) increases, due to the computational complexity of the problem.

\section{NUMERICAL RESULTS}

The simulations were carried out with service areas consisting of 4, 6, 9 and 12 APs and 20, 30, 40 and 50 users, respectively, forming a WLAN. APs are placed 60 meters from each others, 20 meters from adjacent walls and the service area's lengths and widths vary with the number of APs. Transmitted power at each $\mathrm{AP}$ is set to $17 \mathrm{dBm}(50 \mathrm{~mW})$. The receiver detection threshold is assumed to be $-110 \mathrm{dBm}$. In other words, if the user is receiving a signal from an AP that falls below the detection threshold, then this signal is assumed to cause no interference at the receiver. Finally, the receiver sensitivity threshold is assumed to be $-85 \mathrm{dBm}$.

The worst possible scenario would be when all the users are distributed in the overlapping region between APs, Fig. 2. In this case, the best channel assignment leading to the maximum SIR among users is the same as the best channel assignment based on minimum interference between APs. This is obvious due to the fact that users fall in the overlapping coverage region between the two APs. 


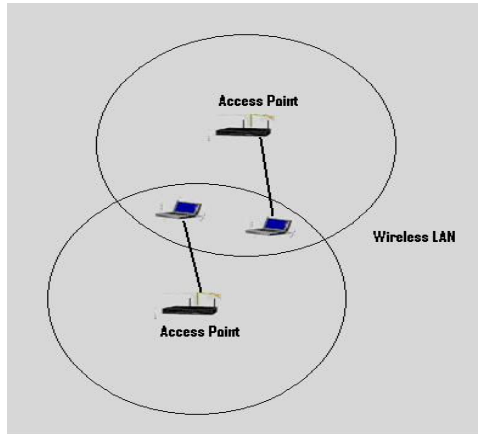

Fig. 2. Users in the overlapping region (worst possible scenario)

On the other hand, the best possible scenario is when the users are in the AP coverage zone but not the overlap region, as shown in Fig. 3. In this case, the best channel assignment based on maximizing the SIR could be any set of combinations of channels since the users are not in the interference region and adjacent channel or co-channel interference has no impact on the users. Therefore, in that case, the same channel can be assigned to all APs. These observations were validated by our algorithm when it was run under these two conditions.

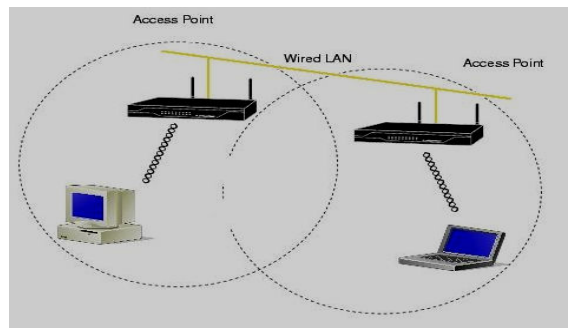

Fig. 3. Users not in the overlapping region (best possible scenario)

\subsection{Simulation Scenario 1}

In scenario 1, we consider a grid of 4 APs over a 100x100 m area and 20 randomly distributed users. We run the model in [6] to get the final transmitted power level at each AP, which in turn leads us to the final received power by the user, and the final association matrix. The final association matrix is the user to AP assignment that leads to the best load distribution. Fig. 4 shows the final user to AP assignment for the scenario under consideration.

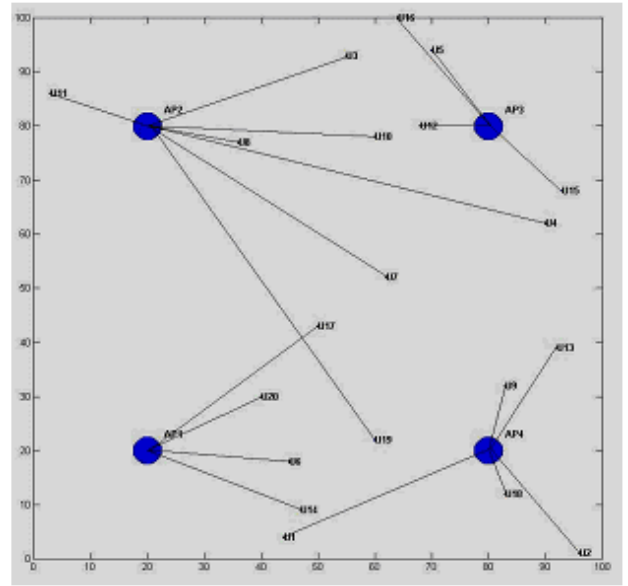

Fig. 4. User to AP association

If we were to look closer at figure 4, we will notice that the user between AP1 and AP4 is associated to AP2 although it is closer to AP1 or AP4. However, this association represents the final association after the power has been decremented on the MCAPs iteratively. Therefore, the final transmitted power at AP1, AP2, AP3 and AP4 is $11 \mathrm{dBm}, 9$ $\mathrm{dBm}, 4 \mathrm{dBm}$, and $3 \mathrm{dBm}$ respectively, and that particular user ended up associating with AP2 leading to better load distribution [6]. Decision has been made based on the powermanagement algorithm presented in [6].

Next, an initial channel assignment is obtained based on minimizing the interference between APs. We invoke model in (3) to find the best channel assignment that leads to the maximum SIR. We apply the initial channel assignment condition at the balanced network with the same power levels achieved by the APs and the corresponding user-to-AP association. Results are shown in Table I. This procedure is followed throughout the other scenarios as well.

TABLE I

COMPARISON BETWEEN OUR MODEL AND MODELS BASED ON MINIMIZING INTERFERENCE BETWEEN APS (SCENARIO 1)

\begin{tabular}{|c|c|c|}
\hline & $\begin{array}{c}\text { Initial Channel } \\
\text { Assignment }\end{array}$ & $\begin{array}{c}\text { Final Channel } \\
\text { Assignment }\end{array}$ \\
\hline AP1 & 11 & 1 \\
\hline AP2 & 1 & 6 \\
\hline AP3 & 8 & 11 \\
\hline AP4 & 3 & 2 \\
\hline Average SIR & 4.48 & 5.83 \\
\hline
\end{tabular}

Table I shows that if we were to start with a channel assignment in the initial design stage and keep that channel assignment unchanged after users are entered into the network, the average SIR of all users would be 4.48 . However, by applying our algorithm at the balanced state, the average SIR was improved by almost $30 \%$ (at 5.83). 


\subsection{Simulation Scenario 2}

In scenario 2, we constructed 6 APs over $160 \mathrm{~m} \mathrm{x} 100 \mathrm{~m}$ and 30 randomly distributed users. We run our model in [6] to get the final transmitted power level at each AP and the final users' association matrix. Table II shows the results for the 6AP scenario.

TABLE II

COMPARISON BETWEEN OUR MODEL AND MODELS BASED ON MINIMIZING INTERFERENCE BETWEEN APs (SCENARIO 2)

\begin{tabular}{|c|c|c|}
\hline & $\begin{array}{c}\text { Initial Channel } \\
\text { Assignment }\end{array}$ & $\begin{array}{c}\text { Final Channel } \\
\text { Assignment }\end{array}$ \\
\hline AP1 & 6 & 2 \\
\hline AP2 & 1 & 11 \\
\hline AP3 & 6 & 6 \\
\hline AP4 & 11 & 6 \\
\hline AP5 & 1 & 8 \\
\hline AP6 & 11 & 1 \\
\hline Average SIR & 2.64 & 2.80 \\
\hline
\end{tabular}

From the above results, we again notice the improvement in the average SIR over all users. The average SIR of all users was improved by almost $6 \%$. In this case, both AP3 and AP4 used channel 6 , which means there were no users in their overlapping region.

\subsection{Simulation Scenario 3}

In this scenario, we deploy 9 APs over $160 \mathrm{~m} \times 160 \mathrm{~m}$ area, where they are distributed in a $3 \times 3$ grid, with 50 users randomly distributed on the service area. Similar procedure is followed as before. Results for this scenario are depicted in Table III.

TABLE III

COMPARISON BETWEEN OUR MODEL AND MODELS BASED ON MINIMIZING INTERFERENCE BETWEEN APS (SCENARIO 3)

\begin{tabular}{|c|c|c|}
\hline \multicolumn{2}{|c|}{$\begin{array}{c}\text { Initial Channel } \\
\text { Assignment }\end{array}$} & $\begin{array}{c}\text { Final Channel } \\
\text { Assignment }\end{array}$ \\
\hline AP1 & 4 & 6 \\
\hline AP2 & 9 & 1 \\
\hline AP3 & 1 & 11 \\
\hline AP4 & 11 & 8 \\
\hline AP5 & 1 & 11 \\
\hline AP6 & 11 & 4 \\
\hline AP7 & 6 & 6 \\
\hline AP8 & 11 & 8 \\
\hline AP9 & 6 & 11 \\
\hline Average SIR & 1.11 & \\
\hline
\end{tabular}

From the displayed results, we can tell that the average SIR of all the users was improved by almost $74 \%$. This improvement can be related to the fact that after load balancing some users that are close to their original AP assignment are now redirected to a farther AP that balances the load. Therefore, it will suffer great interference from its near AP but yet enough RSSI to associate to another AP.

\subsection{Simulation Scenario 4}

Finally, we apply our algorithm on a 12-AP service area. The 12 APs are located as a $3 \times 4$ grid. This time 60 users are generated randomly on the service area. Similar steps are followed for comparison between the channel assignment algorithm based on the minimum interference between APs and our proposed algorithm. Comparison results are recorded in Table IV.

TABLE IV COMPARISON BETWEEN OUR MODEL AND MODELS BASED ON MINIMIZING INTERFERENCE BETWEEN APS

\begin{tabular}{|c|c|c|}
\hline & $\begin{array}{c}\text { Initial Channel } \\
\text { Assignment }\end{array}$ & $\begin{array}{c}\text { Final Channel } \\
\text { Assignment }\end{array}$ \\
\hline AP1 & 1 & 1 \\
\hline $\mathrm{AP} 2$ & 11 & 1 \\
\hline AP3 & 1 & 6 \\
\hline AP4 & 6 & 1 \\
\hline$\overline{\text { AP5 }}$ & 11 & 6 \\
\hline AP6 & 6 & 1 \\
\hline AP7 & 1 & 11 \\
\hline$\overline{\text { AP8 }}$ & 6 & 1 \\
\hline$\overline{\mathrm{AP} 9}$ & 11 & 5 \\
\hline AP10 & 1 & 1 \\
\hline AP11 & 9 & 8 \\
\hline AP12 & 4 & 1 \\
\hline Average SIR & 4.74 & 7.23 \\
\hline
\end{tabular}

We can notice from the above results that our algorithm was efficient in assigning the same channels to APs where there was no overlapping in AP coverages, which caused the average SIR over all users to improve greatly (almost 53\%).

In all scenarios, the NLIP algorithm showed significant improvement in the total SIR, when channel assignment is conducted again at the end of the balanced state. It is important to note, however, that users were distributed randomly in every scenario and it is very hard sometimes to arrange, a priori, the users to be in the overlapping region of all APs.

\section{CONCLUSION}

In this paper, a channel assignment algorithm has been proposed based on maximizing the SIR at the users. The algorithm extends the model presented in [6], where load balancing technique is proposed based on power management, to include channel assignment at the balanced state considering the SIR for users. The algorithm has shown to provide better results compared to previous work where channel assignment was made at an initial stage with no considerations given to users, taking into consideration interference between APs rather than SIR. 
The problem discussed in this paper was developed for research development purposes and not for real-time applications, due to numerous existing complications. The model has proven to perform well for small networks. But due to the computational complexity of the problem defined in (3), future work could involve solving the NLIP by linearizing it. Interested researchers could be guided to a multicriteria optimization formulation after the linearization procedure is executed. This could lead to solving larger size networks efficiently. Upon solving the NLIP on a real time basis, one can include dynamic changes in the user's locations and mobility. In other words, the 8-step algorithm described in Section III would include optimizing over all instances when a user leaves or join a network. This would lead toward operational application of the NLIP model.

\section{REFERENCES}

[1] Y. Lee, K. Kim, and Y. Choi., "Optimization of AP placement and Channel Assignment in Wireless LANs" LCN 2002. 27 $7^{\text {th }}$ Annual IEEE Conference on Local Computer Networks, IEEE Computer Society, Washington D.C. USA, November 2002, pp. 831-836.

[2] Eisenblätter, A., Geerdes, H.-F. and Siomina, I., "Integrated Access Point Placement and Channel Assignment for Wireless LANs in an Indoor Office Environment," 8th IEEE Intl. Symposium on a World of Wireless,
Mobile and Multimedia Networks, June 2007

[3] R. Akl and A. Arepally, "Dynamic Channel Assignment in IEEE 802.11 Networks," Proceedings of IEEE Portable 2007: International Conference on Portable Information Devices, March 2007

[4] R. Rodrigues, G. Mateus, A. Loureiro, "Optimal Base Station Placement and Fixed Channel Assignment Applied to Wireless Local Area Network Projects," Seventh IEEE International Conference on Networks (ICON'99), 1999, pp.186.

[5] A. Mishra, S. Banerjee, and W. Arbaugh, "Weighted Coloring Based Channel Assignment for WLANs," ACM SIGMOBILE Mobile Computing and Communications Review, vol.9, pp.19-31, 2005.

[6] M. Haidar, R. Akl, H. Al-Rizzo, Y. Chan, R. Adada, "Optimal Load Distribution in Large Scale WLAN Networks Utilizing a Power Management Algorithm," Proceedings of IEEE Sarnoff Symposium, May 2007.

[7] M. Boulmalf, H. El-Sayed, and A. Soufyane, "Measured Throughput and SNR of IEEE $802.11 \mathrm{~g}$ in a Small Enterprise Environment," $61^{\text {st }}$ Vehicular IEEE Vehicular Technology Conference, Vol. 2, pp. 1333-1337, Stockholm, Sweden, May 2005.

[8] http://www.draytek.co.uk/support/kb_vigor_wlanchannels.html.

[9] J. Lei, R. Yates, L. Greenstein, and H. Liu, "Wireless Link SNR Mapping Onto An Indoor Testbed," Proceedings of IEEE Tridentcom 2005, pp.130-135, Trento, Italy, Feb. 2005. 